\title{
CHARACTERISTIC FUNCTION AND DEFICIENCY OF ALGEBROID FUNCTIONS ON ANNULI
}

\author{
ASHOK RATHOD
}

\begin{abstract}
In this paper, we develop the value distribution theory for meromorphic functions with maximal deficiency sum for algebroid functions on annuli and we study the relationship between the deficiency of algebroid function on annuli and that of their derivatives. Let $W(z)$ be an $\nu$-valued algebroid function on the annulus $\mathbb{A}\left(\frac{1}{R_{0}}, R_{0}\right)\left(1<R_{0} \leqslant+\infty\right)$ with maximal deficiency sum and the order of $W(z)$ is finite. Then

i. $\limsup _{r \rightarrow \infty} \frac{T_{0}\left(r, W^{\prime}\right)}{T_{0}(r, W)}=2-\delta_{0}(\infty, W)-\theta_{0}(\infty, W)$

ii. $\limsup _{r \rightarrow \infty} \frac{N_{0}\left(r, \frac{1}{W^{\prime}}\right)}{T_{0}\left(r, W^{\prime}\right)}=0$;

iii. $\frac{1-\delta_{0}(\infty, W)}{2-\delta_{0}(\infty, W)} \leqslant K_{0}\left(W^{\prime}\right) \leqslant \frac{2\left(1-\delta_{0}(\infty, W)\right)}{2-\delta_{0}(\infty, W)}$,
\end{abstract}

where

$$
K_{0}\left(W^{\prime}\right)=\limsup _{r \rightarrow \infty} \frac{N_{0}\left(r, W^{\prime}\right)+N_{0}\left(r, \frac{1}{W^{\prime}}\right)}{T_{0}\left(r, W^{\prime}\right)} .
$$

Keywords : Nevanlinna Theory, maximal deficiency sum, algebroid functions, the annuli, etc.

Subject Classification: 30D35

\section{INTRODUCTION}

The uniqueness theory of algebroid functions is an interesting problem in the value distribution theory. The uniqueness problem of algebroid functions was first considered by Valiron, afterwards several uniqueness theorems of algebroid functions in the complex plane $\mathbb{C}$ were proved (see [3],[11]). In 2005, A.Ya. Khrystiyanyn and A.A. Kondratyuk have proposed the Nevanlinna theory for meromorphic functions on annuli (see [4], [5]) and after this work others have done lot of work in this area (see [8], [12], [13]-[36]). In 2009, Cao and Yi [1] studied the uniqueness of meromorphic functions sharing some values on annuli. In 2015, Yang Tan [6], Yang Tan and Yue Wang [7] proved some interesting results on the multiple values and uniqueness of algebroid functions on annuli. Thus, it is interesting to consider the uniqueness problem of algebroid functions in multiply connected domains. By doubly connected mapping theorem [10], each doubly connected domain is conformally equivalent to the annulus $\{z: r<|z|<R\}, 0 \leqslant r<R \leqslant+\infty$. We consider only two cases: $r=0, R=+\infty$ and $0 \leqslant r<R \leqslant+\infty$. In the latter case the homothety $z \mapsto \frac{z}{r R}$ reduces the given domain to the

Ashok Rathod, Characteristic function And Deficiency of Algebroid functions on AnNuli.

(C) AsHoK RATHOD 2019.

The author is supported by the UGC-Rajiv Gandhi National Fellowship (no. F1-17.1/2013-14-SC-KAR40380) of India.

Submitted October 26, $201 \%$. 
annulus $\mathbb{A}\left(\frac{1}{R_{0}}, R_{0}\right)=\left\{z: \frac{1}{R_{0}}<|z|<R_{0}\right\}$, where $R_{0}=\sqrt{\frac{R}{r}}$. Thus, in both cases each annulus is invariant with respect to the inversion $z \mapsto \frac{1}{z}$. We assume that the reader is familiar with the Nevanlinna theory of meromorphic functions and algebroid functions (see [2] and [9]).

Let $A_{v}(z), A_{v-1}(z), \ldots, A_{0}(z)$ be a group of analytic functions which have no common zeros and are defined on the annulus $\mathbb{A}\left(\frac{1}{R_{0}}, R_{0}\right), 1<R_{0} \leqslant+\infty$ and

$$
\psi(z, W)=A_{v}(z) W^{v}+A_{v-1}(z) W^{v-1}+\ldots+A_{1}(z) W+A_{0}(z)=0 .
$$

Then irreducible equation 11 defines a $v$-valued algebroid function on the annulus $\mathbb{A}\left(\frac{1}{R_{0}}, R_{0}\right)$ $\left(1<R_{0} \leqslant+\infty\right)$.

Let $W(z)$ be a $v$-valued algebroid function on the annulus $\mathbb{A}\left(\frac{1}{R_{0}}, R_{0}\right)\left(1<R_{0} \leqslant+\infty\right)$, we use the notations

$$
\begin{aligned}
& m(r, W)=\frac{1}{\nu} \sum_{j=1}^{\nu} m\left(r, w_{j}\right)=\frac{1}{\nu} \sum_{j=1}^{\nu} \frac{1}{2 \pi} \int_{0}^{2 \pi} \log ^{+}\left|w_{j}\left(r e^{i \theta}\right)\right| d \theta, \\
& N_{1}(r, W)=\frac{1}{\nu} \int_{\frac{1}{r}}^{1} \frac{n_{1}(t, W)}{t} d t \\
& N_{2}(r, W)=\frac{1}{\nu} \int_{1}^{r} \frac{n_{2}(t, W)}{t} d t \\
& \bar{N}_{1}\left(r, \frac{1}{W-a}\right)=\frac{1}{\nu} \int_{\frac{1}{r}}^{1} \frac{\bar{n}_{1}\left(t, \frac{1}{W-a}\right)}{t} d t \\
& \bar{N}_{2}\left(r, \frac{1}{W-a}\right)=\frac{1}{\nu} \int_{1}^{r} \frac{\bar{n}_{2}\left(t, \frac{1}{W-a}\right)}{t} d t \\
& \bar{N}_{1}^{k)}\left(r, \frac{1}{W-a}\right)=\frac{1}{\nu} \int_{\frac{1}{r}}^{1} \frac{\bar{n}_{1}^{k)}\left(t, \frac{1}{W-a}\right)}{t} d t, \\
& \bar{N}_{2}^{k)}\left(r, \frac{1}{W-a}\right)=\frac{1}{\nu} \int_{1}^{r} \frac{\bar{n}_{2}^{k)}\left(t, \frac{1}{w-a}\right)}{t} d t, \\
& \bar{N}_{1}^{(k}\left(r, \frac{1}{W-a}\right)=\frac{1}{\nu} \int_{\frac{1}{r}}^{1} \frac{\bar{n}_{1}^{(k}\left(t, \frac{1}{W-a}\right)}{t} d t, \\
& \bar{N}_{2}^{(k}\left(r, \frac{1}{W-a}\right)=\frac{1}{\nu} \int_{1}^{r} \frac{\bar{n}_{2}^{(k}\left(t, \frac{1}{W-a}\right)}{t} d t, \\
& m_{0}(r, W)=m(r, W)+m\left(\frac{1}{r}, W\right)-2 m(1, W), \\
& N_{0}(r, W)=N_{1}(r, W)+N_{2}(r, W) \text {, } \\
& \bar{N}_{0}\left(r, \frac{1}{W-a}\right)=\bar{N}_{1}\left(r, \frac{1}{W-a}\right)+\bar{N}_{2}\left(r, \frac{1}{W-a}\right) \text {, } \\
& \bar{N}_{0}^{k}\left(r, \frac{1}{W-a}\right)=N_{1}^{k}\left(r, \frac{1}{W-a}\right)+N_{2}^{k}\left(r, \frac{1}{W-a}\right),
\end{aligned}
$$

where $w_{j}(z)(j=1,2, \ldots, \nu)$ is a single-valued branch of $W(z), n_{1}(t, W)$ is the counting functions of poles of the function $W(z)$ in $\{z: t<|z| \leqslant 1\}$ and $n_{2}(t, W)$ is the counting functions of poles of the function $W(z)$ in $\{z: 1<|z| \leqslant t\}$ (both counting multiplicity). The symbol $\bar{n}_{1}\left(t, \frac{1}{W-a}\right)$ stands for the counting functions of poles of the function $\frac{1}{W-a}$ in $\{z: t<|z| \leqslant 1\}$ and 
$\bar{n}_{2}\left(t, \frac{1}{W-a}\right)$ is the counting functions of poles of the function $\frac{1}{W-a}$ in $\{z: 1<|z| \leqslant t\}$ (both ignoring multiplicity). By $n_{1}^{k}(t, a, W)$ we denote the number of zeros of $W-a$ in $\{z: t<|z| \leqslant 1\}$ and $n_{2}^{k}(t, a, W)$ is the number of zeros of $W-a$ in $\{z: 1<|z| \leqslant t\}$, where zero of order $<k$ is counted according to its multiplicity and a zero of order $\geqslant k$ is counted exactly $k$ times, respectively.

Let $W(z)$ be a $v$-valued algebroid function defined by 1 on the annulus $\mathbb{A}\left(\frac{1}{R_{0}}, R_{0}\right)$ $\left(1<R_{0} \leqslant+\infty\right)$, as

$$
a \in \mathbb{C}, \quad n_{0}\left(r, \frac{1}{W-a}\right)=n_{0}\left(r, \frac{1}{\psi(z, a)}\right), \quad N_{0}\left(r, \frac{1}{W-a}\right)=\frac{1}{\nu} N_{0}\left(r, \frac{1}{\psi(z, a)}\right) .
$$

In particular, as $a=0$, we have $N_{0}\left(r, \frac{1}{W}\right)=\frac{1}{\nu} N_{0}\left(r, \frac{1}{A_{0}}\right)$ and as $a=\infty$, the identity holds $N_{0}(r, W)=\frac{1}{\nu} N_{0}\left(r, \frac{1}{A_{v}}\right)$. Here $n_{0}\left(r, \frac{1}{W-a}\right)$ and $n_{0}\left(r, \frac{1}{\psi(z, a)}\right)$ are the counting function of zeros of $W(z)-a$ and $\psi(z, a)$ on the annulus $\mathbb{A}\left(\frac{1}{R_{0}}, R_{0}\right)\left(1<R_{0} \leqslant+\infty\right)$, respectively.

Definition 1. [6] Given an algebroid function $W(z)$ on the annulus $\mathbb{A}\left(\frac{1}{R_{0}}, R_{0}\right)$ $\left(1<R_{0} \leqslant+\infty\right)$, the function

$$
T_{0}(r, W)=m_{0}(r, W)+N_{0}(r, W), \quad 1 \leqslant r<R_{0}
$$

is called Nevanlinna characteristic of $W(z)$.

Definition 2. Given an algebroid function $W(z)$ on the annulus $\mathbb{A}\left(\frac{1}{R_{0}}, R_{0}\right)$ $\left(1<R_{0} \leqslant+\infty\right)$, the order of $W(z)$ is defined by

$$
\sigma(W)=\limsup _{r \rightarrow+\infty} \frac{\log T_{0}(r, W)}{\log r}
$$

Definition 3. Given an algebroid function $W(z)$ on the annulus $\mathbb{A}\left(\frac{1}{R_{0}}, R_{0}\right)$ $\left(1<R_{0} \leqslant+\infty\right)$, the value

$$
\delta_{0}(a, W)=\liminf _{r \rightarrow+\infty} \frac{m_{0}\left(r, \frac{1}{W-a}\right)}{T_{0}(r, W)}
$$

is called the deficiency of the function $W(z)$ for the value $a$. For $a=\infty$, we let

$$
\delta_{0}(\infty, W)=\liminf _{r \rightarrow+\infty} \frac{m_{0}(r, W)}{T_{0}(r, W)}=1-\limsup _{r \rightarrow+\infty} \frac{N_{0}(r, W)}{T_{0}(r, W)} .
$$

If $\delta_{0}, a \in \mathbb{C}_{\infty}$, we call a a deficient value of $W(z)$.

Definition 4. Given an algebroid function $W(z)$ on the annulus $\mathbb{A}\left(\frac{1}{R_{0}}, R_{0}\right)$ $\left(1<R_{0} \leqslant+\infty\right)$, the value

$$
\Theta_{0}(a, W)=1-\limsup _{r \rightarrow+\infty} \frac{\bar{N}_{0}\left(r, \frac{1}{W-a}\right)}{T_{0}(r, W)}
$$

and

$$
\theta_{0}(a, W)=\liminf _{r \rightarrow+\infty} \frac{N_{0}\left(r, \frac{1}{W-a}\right)-\bar{N}_{0}\left(r, \frac{1}{W-a}\right)}{T_{0}(r, W)}
$$

are called the reduced deficiencies of the function $W(z)$ for the value a. 


\section{Auxiliary Lemmata}

Lemma 1 (The first fundamental theorem on annuli [7]). Let $W(z)$ be v-valued algebroid function defined by $(1)$ on the annulus $\mathbb{A}\left(\frac{1}{R_{0}}, R_{0}\right)\left(1<R_{0} \leqslant+\infty\right), a \in \mathbb{C}$. Then

$$
T_{0}\left(r, \frac{1}{W-a}\right)=m_{0}\left(r, \frac{1}{W-a}\right)+N_{0}\left(r, \frac{1}{W-a}\right)=T_{0}(r, W)+O(1) .
$$

Lemma 2 (The second fundamental theorem on annuli [13]). Let $W(z)$ be $\nu$-valued algebroid function defined by $(1)$ on the annulus $\mathbb{A}\left(\frac{1}{R_{0}}, R_{0}\right)\left(1<R_{0} \leqslant+\infty\right), a_{k},(k=1,2, . ., p)$ are $p$ distinct complex numbers (finite or infinite), then we have

$$
(p-2 v) T_{0}(r, W) \leqslant \sum_{k=1}^{p} N_{0}\left(r, \frac{1}{W-a_{k}}\right)-N_{1}(r, W)+S_{0}(r, W)
$$

where $N_{1}(r, W)$ is the density index of all multiple values including finite or infinite, every $\tau$-multiple value is counted $\tau-1$ times and

$$
S_{0}(r, W)=m_{0}\left(r, \frac{W^{\prime}}{W}\right)+\sum_{j=1}^{p} m_{0}\left(r, \frac{W^{\prime}}{W-a_{k}}\right)+O(1) .
$$

The remainder satisfies the identity

$$
S_{0}(r, W)=O\left(\log T_{0}(r, W)\right)+O(\log r),
$$

outside a set of a finite linear measure if $r \rightarrow R_{0}=+\infty$, while

$$
S_{0}(r, W)=O\left(\log T_{0}(r, W)\right)+O\left(\log \frac{1}{R_{0}-r}\right),
$$

outside a set $E$ such that

$$
\int_{E} \frac{d r}{R_{0}-r}<+\infty \quad \text { as } \quad r \rightarrow R_{0}<+\infty
$$

Lemma 3. [7] Let $W(z)$ be $\nu$-valued algebroid function defined by (1) on the annulus $\mathbb{A}\left(\frac{1}{R_{0}}, R_{0}\right)\left(1<R_{0} \leqslant+\infty\right)$. If the following conditions are satisfied

$$
\begin{array}{ll}
\liminf _{r \rightarrow \infty} \frac{T_{0}(r, W)}{\log r}<\infty, & R_{0}=+\infty, \\
\liminf _{r \rightarrow R_{0}^{-}} \frac{T_{0}(r, W)}{\log \frac{1}{\left(R_{0}-r\right)}}<\infty, & R_{0}<+\infty,
\end{array}
$$

then $W(z)$ is an algebraic function.

\section{MAIN RESUlTS}

In the present paper we study the problem on the maximal deficiency sum for algebroid function on annuli as well as the relationship between the deficiency of algebroid function on annuli and that of their derivatives.

Theorem 1. Let $W(z)$ be an $\nu$-valued algebroid function defined by (1) on the annulus $\mathbb{A}\left(\frac{1}{R_{0}}, R_{0}\right)\left(1<R_{0} \leqslant+\infty\right)$. Then the set of all numbers $a \in \overline{\mathbb{C}}$ obeying $\Theta_{0}(a, W)>0$ is countable and $\sum_{a \in \overline{\mathbb{C}}} \Theta_{0}(a, W) \leqslant 2$. 
Proof. By the second fundamental theorem for algebroid function on annuli we have

$$
(q-2 \nu) T_{0}(r, W) \leqslant \sum_{i=1}^{q} \bar{N}_{0}\left(r, \frac{1}{W-a_{i}}\right)+S_{0}(r, W)
$$

and this implies

$$
(q-2 \nu) \leqslant \sum_{i=1}^{q} \frac{\bar{N}_{0}\left(r, \frac{1}{W-a_{i}}\right)}{T_{0}(r, W)}+\frac{S_{0}(r, W)}{T_{0}(r, W)} \leqslant \sum_{i=1}^{q} \limsup _{r \rightarrow \infty} \frac{\bar{N}_{0}\left(r, \frac{1}{W-a_{i}}\right)}{T_{0}(r, W)}+\limsup _{r \rightarrow \infty} \frac{S_{0}(r, W)}{T_{0}(r, W)} .
$$

Since

$$
\Theta_{0}(a, W)=1-\limsup _{r \rightarrow \infty} \frac{\bar{N}_{0}\left(r, \frac{1}{W-a i}\right)}{T_{0}(r, W)}
$$

and

$$
S_{0}(r, W)=O\left(T_{0}(r, W)\right)
$$

we have

$$
(q-2 \nu) \leqslant \sum_{i=1}^{q}\left[1-\Theta_{0}\left(a_{i}, W\right)\right] \leqslant q-\sum_{i=1}^{q} \Theta_{0}\left(a_{i}, W\right)
$$

Therefore,

$$
\sum_{i=1}^{q} \Theta_{0}\left(a_{i}, W\right) \leqslant 2 \nu
$$

and this holds for all extended complex numbers as $q \geqslant 3$. Hence,

$$
\sum_{a \in \overline{\mathbb{C}}} \Theta_{0}(a, W) \leqslant 2
$$

and this is a defect relation for algebroid functions on annuli.

Let $E=\{a \in \overline{\mathbb{C}}: \Theta(a, W)>0\}$ and we are going to show that that $E$ is countable set. We denote

$$
E_{n}=\left\{a \in \overline{\mathbb{C}}: \Theta(a, W)>\frac{1}{n}\right\}, n \in \mathbb{N} .
$$

Then, by the defect relation $E_{n}$ contains at most $2 n$ elements and hence $\cup_{n=1}^{\infty} E_{n}$ is a countable set. Let us show that

$$
\cup_{n=1}^{\infty} E_{n}=E .
$$

In order to do this, we take $a \in E$ and hence $\Theta_{0}(a, W)>0$. By the Archimedian property, there exist $n \in \mathbb{N}$ such that

$$
\Theta_{0}(a, W)>\frac{1}{n}
$$

Thus, $a \in E_{n} \subset \cup_{n=1}^{\infty} E_{n}$ and this yields

$$
E \subset \cup_{n=1}^{\infty} E_{n} .
$$

On the other hand, given $a \in \cup_{n=1}^{\infty} E_{n}$, we have $a \in E_{n}$ for some $n \in \mathbb{N}$. Therefore,

$$
\Theta_{0}(a, W)>\frac{1}{n}>0
$$

and hence, $a \in E$. Now we infer that

$$
\cup_{n=1}^{\infty} E_{n} \subset E .
$$

By (3) and (4) we get $E=\cup_{n=1}^{\infty} E_{n}$ and therefore, $E$ is a countable set. 
Remark 1. By Theorem 1, the total deficiency of each algebroid function $W(z)$ on annuli satisfies the inequality

$$
\sum_{a \in \overline{\mathbb{C}}} \delta_{0}(a, W)+\delta_{0}(\infty, W) \leqslant 2 .
$$

If (5) holds, then we say that $W(z)$ has a maximal deficiency sum.

Theorem 2. Let $W(z)$ be an $\nu$-valued algebroid function defined by (1) on the annulus $\mathbb{A}\left(\frac{1}{R_{0}}, R_{0}\right)\left(1<R_{0} \leqslant+\infty\right)$. Then

$$
\limsup _{r \rightarrow \infty} \frac{T_{0}\left(r, W^{\prime}\right)}{T_{0}(r, W)} \leqslant 2 \nu-\delta_{0}(\infty, W)-\delta_{0}(\infty, W) .
$$

Proof. We have

$$
m_{0}\left(r, W^{\prime}\right)=m_{0}\left(r, W \frac{W^{\prime}}{W}\right) \leqslant m_{0}\left(r, \frac{W^{\prime}}{W}\right)+m_{0}(r, W)+O(1) .
$$

By Lemma 2, the identity

$$
m_{0}\left(r, \frac{W^{(k)}}{W}\right)=S_{0}(r, W)
$$

holds true and hence,

$$
m_{0}\left(r, W^{\prime}\right) \leqslant m_{0}(r, W)+S_{0}(r, W) .
$$

We also have

$$
N_{0}\left(r, W^{\prime}\right)=N_{0}(r, W)+\bar{N}_{0}(r, W)+N_{x}(r, W) .
$$

By (6) and (7) we conclude that

$$
\begin{aligned}
T_{0}\left(r, W^{\prime}\right) & =m_{0}\left(r, W^{\prime}\right)+N_{0}\left(r, W^{\prime}\right) \leqslant m_{0}(r, W)+m_{0}\left(r, \frac{W^{\prime}}{W}\right)+N_{0}\left(r, W^{\prime}\right), \\
& \leqslant m_{0}(r, W)+N_{0}(r, W)+\bar{N}_{0}(r, W)+N_{x}(r, W)+S_{0}(r, W) \\
& \leqslant T_{0}(r, W)+\bar{N}_{0}(r, W)+N_{x}(r, W)+S_{0}(r, W) .
\end{aligned}
$$

This yields

$$
\frac{T_{0}\left(r, W^{\prime}\right)}{T_{0}(r, W)} \leqslant 1+\frac{\bar{N}_{0}(r, W)}{T_{0}(r, W)}+\frac{N_{x}(r, W)}{T_{0}(r, W)}+\frac{S_{0}(r, W)}{T_{0}(r, W)} .
$$

Therefore,

$$
\begin{aligned}
\limsup _{r \rightarrow \infty} \frac{T_{0}\left(r, W^{\prime}\right)}{T_{0}(r, W)} & \leqslant 1+\limsup _{r \rightarrow \infty} \frac{\bar{N}_{0}(r, W)}{T_{0}(r, W)} \\
& \leqslant 1+1-\Theta_{0}(\infty, W) \leqslant 2-\Theta_{0}(\infty, W)
\end{aligned}
$$

But

$$
\delta_{0}(\infty, W)+\theta_{0}(\infty, W) \leqslant \Theta_{0}(\infty, W)
$$

and hence,

$$
\limsup _{r \rightarrow \infty} \frac{T_{0}\left(r, W^{\prime}\right)}{T_{0}(r, W)} \leqslant 2-\delta_{0}(\infty, W)-\theta_{0}(\infty, W) .
$$

The proof is complete.

Theorem 2 yields the following corollary. 
Corollary 1. Let $W(z)$ be an $\nu$-valued algebroid function defined by (1) on the annulus $\mathbb{A}\left(\frac{1}{R_{0}}, R_{0}\right)\left(1<R_{0} \leqslant+\infty\right)$. Then

$$
\limsup _{r \rightarrow \infty} \frac{T_{0}\left(r, W^{\prime}\right)}{T_{0}(r, W)} \leqslant 2 \nu-\delta_{0}(\infty, W)-\delta_{0}(\infty, W) .
$$

Theorem 3. Let $W(z)$ be an $\nu$-valued algebroid function defined by (1) on the annulus $\mathbb{A}\left(\frac{1}{R_{0}}, R_{0}\right)\left(1<R_{0} \leqslant+\infty\right)$ with a maximal deficiency sum and of a finite order. Then i. $\limsup _{r \rightarrow \infty} \frac{T_{0}\left(r, W^{\prime}\right)}{T_{0}(r, W)}=2-\delta_{0}(\infty, W)-\theta_{0}(\infty, W)$;

ii. $\limsup _{r \rightarrow \infty} \frac{N_{0}\left(r, \frac{1}{W^{\prime}}\right)}{T_{0}\left(r, W^{\prime}\right)}=0$;

iii. $\frac{\substack{r \rightarrow \infty \\ 1-\delta_{0}(\infty, W)}}{2-\delta_{0}(\infty, W)} \leqslant K_{0}\left(W^{\prime}\right) \leqslant \frac{2\left(1-\delta_{0}(\infty, W)\right)}{2-\delta_{0}(\infty, W)}$, where

$$
K_{0}\left(W^{\prime}\right)=\limsup _{r \rightarrow \infty} \frac{N_{0}\left(r, W^{\prime}\right)+N_{0}\left(r, \frac{1}{W^{\prime}}\right)}{T_{0}\left(r, W^{\prime}\right)} .
$$

Proof. We have

$$
\begin{aligned}
T_{0}\left(r, W^{\prime}\right) & =m_{0}\left(r, W^{\prime}\right)+N_{0}\left(r, W^{\prime}\right) \leqslant m_{0}(r, W)+m_{0}\left(r, \frac{W^{\prime}}{W}\right)+N_{0}\left(r, W^{\prime}\right) \\
& \leqslant m_{0}(r, W)+N_{0}(r, W)+\bar{N}_{0}(r, W)+N_{x}(r, W)+S_{0}(r, W) \\
& \leqslant m_{0}(r, W)+N_{0}(r, W)+\bar{N}_{0}(r, W)+2(\nu-1) T_{0}(r, W)+S_{0}(r, W) \\
& \leqslant(2 \nu-1) T_{0}(r, W)+\bar{N}_{0}(r, W)+S_{0}(r, W) \leqslant 2 \nu T_{0}(r, W)+S_{0}(r, W) .
\end{aligned}
$$

By Lemma 2 we get

$$
S_{0}\left(r, W^{(k)}\right)=O\left(\log r T_{0}\left(r, W^{(k)}\right)\right)=O\left(\log r T_{0}(r, W)\right)=S_{0}(r, W)
$$

and hence

$$
m_{0}\left(r, \frac{W^{(k)}}{W-a^{[i]}}\right)=S_{0}(r, W)
$$

holds for each positive $a^{[i]}$. We let

$$
F(z)=\sum_{i=1}^{p} \frac{1}{W(z)-a^{[i]}} .
$$

Then, as in [11], we have

$$
\begin{aligned}
m(r, F)+O(1) & \geqslant \sum_{i=1}^{p} m\left(r, \frac{1}{W(z)-a^{[i]}}\right), \\
m\left(\frac{1}{r}, F\right) & \geqslant \sum_{i=1}^{p} m\left(r, \frac{1}{W(z)-a^{[i]}}\right) .
\end{aligned}
$$

In fact, 10 holds if $p=1$. If $p \geqslant 2$, we let

$$
\delta=\min _{i \neq j}\left|a^{[i]}-a^{[j]}\right| .
$$

It is Obvious that $\delta>0$. Given a fixed $z$, there exist $k \in\{1,2, \ldots, \nu\}$ and $i \in\{1,2, \ldots, q\}$ such that

and the inequality

$$
\left|w_{k}-a^{[i]}\right|<\frac{\delta}{2 q} \leqslant \frac{\delta}{4}
$$

$$
\left|w_{k}(z)-a^{[j]}\right| \geqslant\left|a^{[i]}-a^{[j]}\right|-\left|w_{k}(z)-a^{[i]}\right| \geqslant \frac{3 \delta}{4}
$$


holds true for $i \neq j$. Therefore, the set of points in $\partial \mathbb{C}_{r}$, where $\mathbb{C}_{r}=\{z:|z|=r\}(r=r$ or $r=$ $\left.\frac{1}{r}\right)$ obeying $\left|w_{k}(z)-a^{[i]}\right|<\frac{\delta}{2 q}$ is either empty or each two such sets are mutually disjoint for different $i$. In any case

$$
\begin{aligned}
\frac{1}{2 \pi} \int_{0}^{2 \pi} \log ^{+}\left|F\left(r e^{i \theta}\right)\right| d \theta & \geqslant \frac{1}{2 \pi} \sum_{i=1}^{q} \int_{\left|w_{k}-a^{[i]}\right|<\frac{\delta}{2 q},|z|=r} \log ^{+}\left|F\left(r e^{i \theta}\right)\right| d \theta \\
& \geqslant \frac{1}{2 \pi} \sum_{i=1}^{q} \int_{\left|w_{k}-a^{[i]}\right|<\frac{\delta}{2 q},|z|=r} \log ^{+} \frac{1}{\left|w_{k}\left(r e^{i \theta}\right)-a^{[i]}\right|} d \theta .
\end{aligned}
$$

Since

$$
\begin{aligned}
& \frac{1}{2 \pi} \sum_{i=1}^{q} \int_{\left|w_{k}-a^{[i]}\right|<\frac{\delta}{2 q},|z|=r} \log ^{+} \frac{1}{\left|w_{k}\left(r e^{i \theta}\right)-a^{[i]}\right|} d \theta \\
& \quad=m\left(r, \frac{1}{W(z)-a^{[i]}}\right) \frac{1}{2 \pi} \sum_{i=1}^{q} \int_{\left|w_{k}-a^{[i]}\right| \geqslant \frac{\delta}{2 q},|z|=r} \log ^{+} \frac{1}{\left|w_{k}\left(r e^{i \theta}\right)-a^{[i]}\right|} d \theta \\
& \quad \geqslant m\left(r, \frac{1}{W(z)-a^{[i]}}\right)-q \log ^{+} \frac{2 q}{\delta},
\end{aligned}
$$

we obtain that

$$
\begin{aligned}
m(r, F) & =\frac{1}{\nu} \sum_{k=1}^{\nu} \frac{1}{2 \pi} \int_{0}^{2 \pi} \log ^{+}\left|F\left(r e^{i \theta}\right)\right| d \theta \geqslant \frac{1}{\nu} \sum_{k=1}^{\nu} \sum_{i=1}^{q} m\left(r, \frac{1}{W(z)-a^{[i]}}\right)-\frac{1}{\nu} \log ^{+} \frac{2 q}{\delta} \\
& =\sum_{i=1}^{q} m\left(r, \frac{1}{W(z)-a^{[i]}}\right)-\frac{1}{\nu} \log ^{+} \frac{2 q}{\delta} .
\end{aligned}
$$

Now relation 10 follows the above inequality in the case $r=r$ and $r=\frac{1}{r}$. Since

$$
\begin{aligned}
m(r, F) & =m\left(r, W^{(k)} F\right)+m\left(r, \frac{1}{W^{(k)}}\right) \\
& \leqslant \sum_{i=1}^{p} m\left(r, \frac{W^{(k)}}{W-a^{[i]}}\right)+m\left(r, \frac{1}{W^{(k)}}\right)
\end{aligned}
$$

and

$$
\begin{aligned}
m\left(\frac{1}{r}, F\right) & =m\left(\frac{1}{r}, W^{(k)} F\right)+m\left(\frac{1}{r}, \frac{1}{W^{(k)}}\right) \\
& \leqslant \sum_{i=1}^{p} m\left(\frac{1}{r}, \frac{W^{(k)}}{W-a^{[i]}}\right)+m\left(\frac{1}{r}, \frac{1}{W^{(k)}}\right),
\end{aligned}
$$

we get

$$
m_{0}(r, F) \leqslant \sum_{i=1}^{p} m_{0}\left(r, \frac{W^{(k)}}{W-a^{[i]}}\right)+m_{0}\left(r, \frac{1}{W^{(k)}}\right) .
$$

It follows from (8), (11) and Lemma 3.1 that

$$
\begin{aligned}
\sum_{i=1}^{p} m_{0}\left(r, \frac{1}{W(z)-a^{[i]}}\right) & \leqslant m_{0}\left(r, \frac{1}{W^{(k)}}\right)+S_{0}(r, W) \\
& \leqslant T_{0}\left(r, W^{(k)}\right)-N_{0}\left(r, \frac{1}{W^{(k)}}\right)+S_{0}(r, W) .
\end{aligned}
$$


Thus,

$$
p T_{0}(r, W) \leqslant \sum_{i=1}^{p} N_{0}\left(r, \frac{1}{W(z)-a^{[i]}}\right)+T_{0}\left(r, W^{(k)}\right)-N_{0}\left(r, \frac{1}{W^{(k)}}\right)+S_{0}(r, W) .
$$

By (13) we obtain

$$
p T_{0}(r, W) \leqslant \sum_{i=1}^{p} N_{0}\left(r, \frac{1}{W(z)-a^{[i]}}\right)+T_{0}\left(r, W^{\prime}\right)-N_{0}\left(r, \frac{1}{W^{\prime}}\right)+S_{0}(r, W) .
$$

and hence,

$$
\begin{aligned}
p & \leqslant \liminf _{r \rightarrow \infty} \frac{T_{0}\left(r, W^{\prime}\right)}{T_{0}(r, W)}+\sum_{i=1}^{p}\left(1-\delta_{0}\left(a^{[i]}, W\right)\right)+\liminf _{r \rightarrow \infty} \frac{S_{0}(r, W)}{T_{0}(r, W)} \\
& =p+\limsup _{r \rightarrow \infty} \frac{T_{0}\left(r, W^{\prime}\right)}{T_{0}(r, W)}-\sum_{i=1}^{p} \delta_{0}\left(a^{[i]}, W\right) .
\end{aligned}
$$

Therefore, we have

$$
\sum_{i=1}^{p} \delta_{0}\left(a^{[i]}, W\right) \leqslant \limsup _{r \rightarrow \infty} \frac{T_{0}\left(r, W^{\prime}\right)}{T_{0}(r, W)}
$$

Hence, identity (8) and Lemma 2 imply that

$$
\limsup _{r \rightarrow \infty} \frac{T_{0}\left(r, W^{\prime}\right)}{T_{0}(r, W)} \leqslant 2 \nu-\delta_{0}(\infty, W) .
$$

As $p$ is arbitrary, we combine $(17)$ and $(16)$ to have

$$
2 \nu-\delta_{0}(\infty, W) \leqslant \liminf _{r \rightarrow \infty} \frac{T_{0}\left(r, W^{\prime}\right)}{T_{0}(r, W)} \leqslant \limsup _{r \rightarrow \infty} \frac{T_{0}\left(r, W^{\prime}\right)}{T_{0}(r, W)} \leqslant 2 \nu-\delta_{0}(\infty, W),
$$

that is,

$$
\lim _{r \rightarrow \infty} \frac{T_{0}\left(r, W^{\prime}\right)}{T_{0}(r, W)} \leqslant 2 \nu-\delta_{0}(\infty, W) .
$$

Given $\varepsilon>0$, we choose $q$ sufficiently large so that

$$
\sum_{i=1}^{p} \delta_{0}\left(a^{[i]}, W\right)>2 \nu-\delta_{0}(\infty, W)-\varepsilon .
$$

For these $q$, inequality 12 implies

$$
\begin{aligned}
\limsup _{r \rightarrow \infty} \frac{N_{0}\left(r, \frac{1}{W^{\prime}}\right)}{T_{0}\left(r, W^{\prime}\right)} & +\liminf _{r \rightarrow \infty} \frac{T_{0}(r, W)}{T_{0}\left(r, W^{\prime}\right)} \sum_{i=1}^{p} \liminf _{r \rightarrow \infty} \frac{m_{0}\left(r, \frac{1}{W(z)-a^{[i]}}\right)}{T_{0}(r, W)} \\
& \leqslant 1+\limsup _{r \rightarrow \infty} \frac{S_{0}(r, W)}{T_{0}\left(r, W^{\prime}\right)} .
\end{aligned}
$$

Thus, from 18)-20 we deduce

$$
\limsup _{r \rightarrow \infty} \frac{N_{0}\left(r, \frac{1}{W^{\prime}}\right)}{T_{0}\left(r, W^{\prime}\right)} \leqslant \frac{\varepsilon}{2 \nu-\delta_{0}(\infty, W)} .
$$

Since $\varepsilon>0$ is arbitrary, we have

$$
\limsup _{r \rightarrow \infty} \frac{N_{0}\left(r, \frac{1}{W^{\prime}}\right)}{T_{0}\left(r, W^{\prime}\right)}=0
$$


And since $N_{0}\left(r, W^{\prime}\right) \leqslant 2 \nu N_{0}(r, W)$, we get

$$
\frac{N_{0}\left(r, W^{\prime}\right)}{T_{0}\left(r, W^{\prime}\right)} \frac{T_{0}\left(r, W^{\prime}\right)}{T_{0}(r, W)} \leqslant 2 \nu \frac{N_{0}(r, W)}{T_{0}(r, W)} .
$$

Now it follows from $(18)$ and $(23)$ that

$$
\left(2 \nu-\delta_{0}(\infty, W)\right) \limsup _{r \rightarrow \infty} \frac{N_{0}\left(r, W^{\prime}\right)}{T_{0}\left(r, W^{\prime}\right)} \leqslant 2 \nu\left(1-\delta_{0}(\infty, W)\right) .
$$

Relations (22) and 24) imply

$$
\limsup _{r \rightarrow \infty} \frac{N_{0}\left(r, W^{\prime}\right)+N_{0}\left(r, \frac{1}{W^{\prime}}\right)}{T_{0}\left(r, W^{\prime}\right)} \leqslant \frac{2 \nu\left(1-\delta_{0}(\infty, W)\right)}{\left(2 \nu-\delta_{0}(\infty, W)\right)} .
$$

Therefore, we obtain

$$
K_{0}\left(W^{\prime}\right) \leqslant \frac{2 \nu\left(1-\delta_{0}(\infty, W)\right)}{\left(2 \nu-\delta_{0}(\infty, W)\right)}
$$

Furthermore, we have $N_{0}(r, W) \leqslant N_{0}\left(r . W^{\prime}\right)$. By (18) we infer that

$$
\frac{N_{0}(r, W)}{T_{0}(r, W)} \leqslant\left(2 \nu-\delta_{0}(\infty, W)\right) \frac{N_{0}\left(r, W^{\prime}\right)}{T_{0}\left(r, W^{\prime}\right)} .
$$

Thus,

$$
\limsup _{r \rightarrow \infty} \frac{N_{0}\left(r, W^{\prime}\right)}{T_{0}\left(r, W^{\prime}\right)} \geqslant \frac{1}{\left(2 \nu-\delta_{0}(\infty, W)\right)} \limsup _{r \rightarrow \infty} \frac{N_{0}(r, W)}{T_{0}(r, W)}=\frac{1-\delta_{0}(\infty . W)}{2 \nu-\delta_{0}(\infty, W)}
$$

Hence,

$$
K_{0}\left(W^{\prime}\right) \leqslant \frac{1-\delta_{0}(\infty . W)}{2 \nu-\delta_{0}(\infty, W)}
$$

Due to (25) and (26) we have

$$
\frac{1-\delta_{0}(\infty . W)}{2 \nu-\delta_{0}(\infty, W)} \leqslant K_{0}\left(W^{\prime}\right) \leqslant \frac{2 \nu\left(1-\delta_{0}(\infty, W)\right)}{\left(2 \nu-\delta_{0}(\infty, W)\right)} .
$$

Theorem 4. Let $W(z)$ be an $\nu$-valued algebroid function defined by (1) on the annulus $\mathbb{A}\left(\frac{1}{R_{0}}, R_{0}\right)\left(1<R_{0} \leqslant+\infty\right)$ of finite order and $\delta_{0}(\infty, W)=1$. Then

$$
\sum_{a \in \mathbb{C}} \delta_{0}(a, W) \leqslant \delta_{0}\left(0, W^{\prime}\right)
$$

Proof. If

$$
\sum_{a \in \mathbb{C}} \delta_{0}(a, W)=0
$$

Theorem 3 is valid in this case. In what follows we assume that $\sum_{a \in \mathbb{C}} \delta_{0}(a, W)>0$. Let $\left\{a_{\mu}\right\}$ be a sequence of distinct complex complex numbers in $\mathbb{C}$ containing all the finite deficient values of $W(z)$. For each positive integer $q$ the inequality

$$
\sum_{\mu=1}^{q} m_{0}\left(r, \frac{1}{W(z)-a_{\mu}}\right)+N_{0}\left(r, \frac{1}{W^{\prime}}\right) \leqslant T_{0}\left(r, W^{\prime}\right)+S_{0}(r, W)
$$


holds for any $q$ finite complex numbers in $a_{\mu}$. Therefore, we have

$$
\frac{N_{0}\left(r, \frac{1}{W^{\prime}}\right)}{T_{0}\left(r, W^{\prime}\right)}+\frac{T_{0}(r, W)}{T_{0}\left(r, W^{\prime}\right)}\left(\frac{\sum_{\mu=1}^{q} m_{0}\left(r, \frac{1}{W(z)-a_{\mu}}\right)}{T_{0}(r, W)}-o(1)\right) \leqslant 1,
$$

as $r \rightarrow \infty$. Hence, by inequality (18) we obtain that

$$
\begin{aligned}
& 1 \geqslant \limsup _{r \rightarrow \infty}\left[\frac{N_{0}\left(r, \frac{1}{W^{\prime}}\right)}{T_{0}\left(r, W^{\prime}\right)}+\frac{T_{0}(r, W)}{T_{0}\left(r, W^{\prime}\right)}\left(\frac{\sum_{\mu=1}^{q} m_{0}\left(r, \frac{1}{W(z)-a_{\mu}}\right)}{T_{0}(r, W)}-o(1)\right]\right] \\
& \geqslant \limsup _{r \rightarrow \infty} \frac{N_{0}\left(r, \frac{1}{W^{\prime}}\right)}{T_{0}\left(r, W^{\prime}\right)}+\liminf _{r \rightarrow \infty} \frac{T_{0}(r, W)}{T_{0}\left(r, W^{\prime}\right)}\left(\frac{\sum_{\mu=1}^{q} m_{0}\left(r, \frac{1}{W(z)-a_{\mu}}\right)}{T_{0}(r, W)}-o(1)\right) \\
& \geqslant \limsup _{r \rightarrow \infty} \frac{N_{0}\left(r, \frac{1}{W^{\prime}}\right)}{T_{0}\left(r, W^{\prime}\right)}+\liminf _{r \rightarrow \infty} \frac{T_{0}(r, W)}{T_{0}\left(r, W W^{\prime}\right)} \liminf _{r \rightarrow \infty} \frac{\sum_{\mu=1}^{q} m_{0}\left(r, \frac{1}{W(z)-a_{\mu}}\right)}{T_{0}(r, W)} \\
& \geqslant \limsup _{r \rightarrow \infty} \frac{N_{0}\left(r, \frac{1}{W^{\prime}}\right)}{\sum_{0}\left(r, W^{\prime}\right)}+\frac{q=1}{2 \nu-\delta_{0}\left(a_{\mu}, W\right)} .
\end{aligned}
$$

Since $q$ is arbitrary and $\delta_{0}(\infty, W)=1$, we have

$$
\sum_{a \in \mathbb{C}} \delta_{0}(a, W) \leqslant \delta_{0}\left(0, W^{\prime}\right)
$$

\section{BIBLIOGRAPHY}

1. E. Ullrich. Über den Einfluß der verzweigtheit einer algebloide auf ihre wertvertellung // J. Reine Angew. Math. 1932:167, 198-220 (1932).

2. G. Valiron. Sur quelques propriétés des fonctions algébroides // Compt. Rend. Math. 189, 824826 (1929).

3. N. Baganas. Sur les valeurs algébriques d'une fonctions algebroldes et les intégrales pseudoabelinnes // Annales École Norm. Sup. Sér. III. 66, 161-208 (1949).

4. M. L. Fang. A note on a result of Singh and Kulkarni // Int. J. Math. Sci. 23:4, 285-288 (2000).

5. S.K. Singh, V.N. Kulkarni. Characteristic function of a meromorphic function and its derivative // Ann Polon Math. 28, 123-133 (1973).

6. Yu-Zan He and Ye-Zhou Li. Some results on algebroid functions// Compl. Variab. Theory Appl. 43:3-4, 299-313 (2001).

7. S. Daochun, G. Zongsheng. On the operation of algebroid functions // Acta Math. Sci. 30:1, 247-256 (2010).

8. S. Daochun, G. Zongsheng. Value disribution theory of algebroid functions. Science Press, Beijing (2014).

9. Yu-Zan He and Xiu-Zhi Xiao. Algebroid functions and Ordinarry Difference Equations. Science Press, Beijing (1988).

10. S. Daochun, G. Zongsheng. Theorems for algebroid functions // Acta Math. Sinica. 49:5, 1-6 (2006).

11. Meili Liang. On the value distribution of algebroid functions // SOP Trans. Appl. Math. 1:1, (2014). 
12. W.K. Hayman. Meromorphic functions. Oxford University Press, Oxford (1964).

13. F. Minglang. Unicity theorem for algebroid functions // Acta. Math. Sinica. 3:6, 217-222 (1993).

14. Pingyuan Zhang, Peichu Hu. On uniqueness for algebroid functions of finite order // Acta. Math. Sinica. 35:3, 630-638 (2015).

15. G.S. Prokoporich. Fix-points of meromorphic or entire functions // Ukrainian Math. J. 25:2, 248-260 (1973).

16. Z. Qingcai. Uniqueness of algebroid functions // Math. Pract. Theory. 43:1, 183-187 (2003).

17. Cao Tingbin, Yi Hongxun. On the uniqueness theory of algebroid functions // Southest Asian Bull. Math. 33:1, 25-39 (2009).

18. Zu-Xing Xuan, ZongG-Sheng Gao. Uniqueness theorems for algebroid functions // Compl. Variab. Ellipt. Equat. 51:7, 701-712 (2006).

19. C.C. Yang, H.X. Yi. Uniqueness theory of meromorphic functions. Math. Appl. 557. Kluwer Academic Publishers, Dordrecht (2003).

20. Zhaojun Wu, Sheng'an Chen. Characteristic function and deficiency of meromorphic functions in the punctured plane// Acta Math. Scientia. 35:B(3), 673-680 (2015).

21. R. S. Dyavanal, Ashok Rathod. Uniqueness theorems for meromorphic functions on annuli// Indian J. Math. Math. Sci. 12:1, 1-10 (2016).

22. R. S. Dyavanal, Ashok Rathod. Multiple values and uniqueness of meromorphic functions on annuli // Int. J. Pure Appl. Math. 107:4, 983-995 (2016).

23. R. S. Dyavanal, Ashok Rathod. On the value distribution of meromorphic functions on annuli // Indian J. Math. Math. Sci. 12:2, 203-217 (2016).

24. R.S. Dyavanal, Ashok Rathod. Some generalisation of Nevanlinna's five-value theorem algebroid functions on annuli // Asian J. Math. Comp. Resear. 20:2, 85-95 (2017).

25. R.S. Dyavanal, Ashok Rathod. Nevanlinna's five-value heorem for derivatives of meromorphic functions sharing values on annuli // Asian J. Math. Comp. Resear. 20:1, 13-21 (2017).

26. R. S. Dyavanal, Ashok Rathod. Unicity theorem for algebroid functions related to multiple values and derivatives on annuli// Int. J. Fuzzy Math. Archive. 13:1, 25-39 (2017).

27. R. S. Dyavanal, Ashok Rathod. General Milloux inequality for algebroid functions on annuli // Int. J. Math. Appl. 5:3, 319-326 (2017).

28. Ashok Rathod. The multiple values of algebroid functions and uniqueness // Asian J. Math. Comp. Resear. 14:2, 150-157 (2016).

29. Ashok Rathod. The uniqueness of meromorphic functions concerning five or more values and small functions on annuli // Asian J. Current Res. 1:3, 101-107 (2016).

30. Ashok Rathod. Uniqueness of algebroid functions dealing with multiple values on annuli// J. Basic Appl. Res. Int. 19:3, 157-167 (2016).

31. Ashok Rathod. On the deficiencies of algebriod functions and their differential polynomials // J. Basic Appl. Resear. Int. 1:1, 1-11 (2016).

32. Ashok Rathod. The multiple values of algebroid functions and uniqueness on annuli // Konoralf J. Math. 5:2, 216-227 (2017).

33. Ashok Rathod. Several uniqueness theorems for algebroid functions // J. Anal. 25:2, 203-213 (2017).

34. Ashok Rathod. Nevanlinna's five-value theorem for algebroid functions // Ufa Math. J. 10:2, 127-132 (2018).

35. Ashok Rathod. Nevanlinna's five-value theorem for derivatives of algebroid functions on annuli // Tamkang J. Mathe. 49:2, 129-142 (2018).

36. S. S. Bhoosnurmath, R. S. Dyavanal, Mahesh Barki, Ashok Rathod. Value distribution for nth difference operator of meromorphic functions with maximal deficiency sum // J. Anal. 1-15 (2018).

Ashok Rathod,

Department of Mathematics,

Karnatak University,

Dharwad-580003, India

E-mail: ashokmrmaths@gmail .com 